\title{
Extraction of Polyphenols in Hop Bract Part Discharged from Beer Breweries
}

\author{
Hideki YAMAMOTO ${ }^{1}$, Chika NAGANO ${ }^{1}$, \\ Fumiko TAKEUCHI ${ }^{1}$, Junji SHIBATA ${ }^{1}$, \\ Motoyuki TAGASHIRA ${ }^{2}$ and Yasuyuki OHTAKE ${ }^{2}$ \\ ${ }^{1}$ Department of Chemical Engineering, Kansai University, \\ 3-35, Yamatecho 3, Suita-shi, Osaka 564-8680, Japan \\ ${ }^{2}$ Department of Fundamental Research Laboratory, \\ Asahi Breweries, Ltd., 1-21, Midori 1, Moriya-shi, \\ Ibaragi 302-0106, Japan
}

Keywords: Polyphenol, Catechin, Procyanidin, Extraction, Antioxidative Property

\begin{abstract}
Wastes discharged from beer breweries are mainly composed of malt and hop. The nutritional value of malt is quite high and, consequently, it is recycled as feed for domestic animals; however, the nutritional value of hop as feed is not so high. A new utilization method for hop waste is therefore required. In this experiment, it is found that Procyanidin, a polymer composed of catechins, is abundantly contained in hop bract part and hop itself. This research deals with the extraction of total polyphenols, catechin, and procyanidin from hop bract part under various extraction conditions. When ethanol was used as the extract solvent, about $30 \%$ of the total extracted polyphenols was procyanidin, showing that ethanol has the highest selectivity for procyanidin. The extraction of all polyphenols, catechin, and procyanidin was conducted in about $10 \mathrm{~min}$ at a temperature of $293-333 \mathrm{~K}$ and with horizontal shaking at $130 \mathrm{rpm}$. The amount of each polyphenol extracted increased with increasing temperature. The total amount of catechin extracted reached a maximum at an extraction temperature of $333 \mathrm{~K}$. Compared with tea, more polyphenols were shown to be contained in the hop bract part. A continuous extraction process for collection of the polyphenols contained in the hop bract part using a modified mixer-settler extractor device is described and the fundamental performance of the presented extractor was examined.
\end{abstract}

\section{Introduction}

In May 2001, the food recycling law went into effect for the purpose of recycling and decreasing the quantity of food waste produced in Japan. The main discharge from the beer brewing industry is malt and hop bract part, which total approximately 900,000 t every year. Since the raw materials used in beer production are agricultural crops (malt and hop), recycling has been attempted (Yamamoto et al., 2005a). Because of its high nutritional value, malt waste is recycled as feed for farm animals such as cows and pigs; however, since the hop bract part is less nutritional as feed, a new recycling method is required (Yamamoto et al., 2005b).

Hop and the hop bract part contain large amounts of polyphenols, which have strong antioxidative properties (Kanner et al., 1994). Especially, procyanidin B-2, B-3, and C-2, polymer structures composed of catechin monomers, are known to be abundant (Ariga et al., 1988). The chemical structure of catechin, a flavonoid in the form of a low molecular weight

Received on December 24, 2005. Correspondence concerning this article should be addressed to H. Yamamoto (E-mail address: yhideki@kansai-u.ac.jp). polyphenol with the structure of flavan-3-ol, is shown in Figure 1(a). Catechin, which is also contained in green tea, has a characteristic bitter and astringent taste (Guyot et al., 1998). The chemical structure of procyanidin, a condensed polyphenol composed of polymerized catechin, is shown in Figure 1(b) (Suematsu et al., 1995).

It has been reported that procyanidins have stronger antioxidative properties than catechin, and recently, they have received much attention as a natural antioxidant. In this research, the extraction characteristics of polyphenols contained in hop bract part are measured using several solvents (methanol, ethanol, 1-propanol and water), respectively. In addition, a mixer-settler extractor designed and manufactured to extract these polyphenols is presented. The characteristics of the mixer-settler extractor are also examined under several experimental conditions

\section{Experimental Methods}

1.1 Extraction of polyphenols from hop bract part A photograph of the hop bract part used in this research is shown in Figure 2; the hop bract part was supplied by Asahi Breweries, Ltd. In the extraction 
<smiles>[R20]Oc1cc(O)cc2c1C[C@H]([R10])[C@H](c1ccc(O)c(O)c1)O2</smiles>

$\mathrm{R}_{1}=\mathrm{R}_{2}=\mathrm{H}:(-)$-epicatechin

$\mathrm{R}_{1}=$ galloyl, $\mathrm{R}_{2}=\mathrm{H}$ : (-)-epicatechin-3-gallate

$\mathrm{R}_{1}=\mathrm{R}_{2}=$ galloyl : (-)-epicatechin-3,5-digallate<smiles>[R20]Oc1cc(O)cc2c1C[C@H]([14OH])[C@H](c1cc(O)c(O)c(O)c1)O2</smiles>

$\mathrm{R}_{1}=\mathrm{R}_{2}=\mathrm{H}:(-)$-epigallocatechin

$R_{1}=$ galloyl, $R_{2}=H:(-)$-epigallocatechin-3-gallate

$R_{1}=R_{2}=$ galloyl : (-)-epigallocatechin-3,5-digallate<smiles>N#CC(=O)c1cc(O)c(O)c(O)c1</smiles>

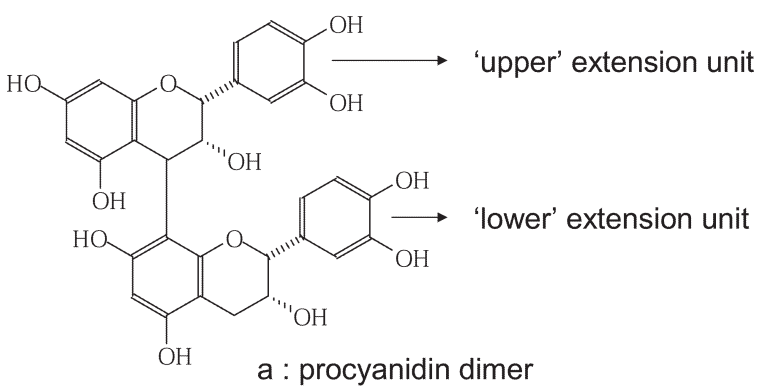

a : procyanidin dimer

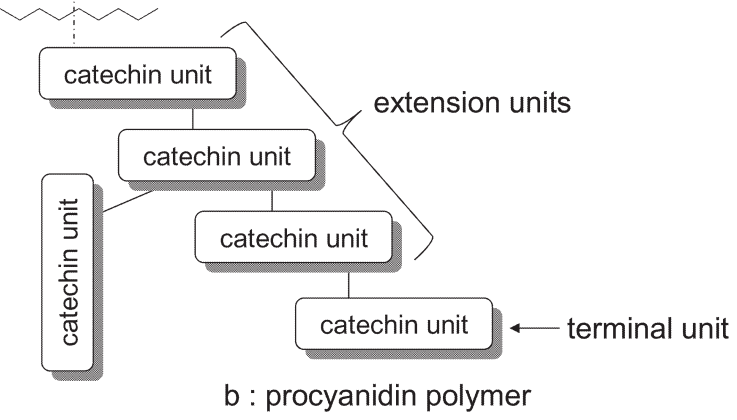

Fig. 1 Chemical structures of catechin and procyanidin

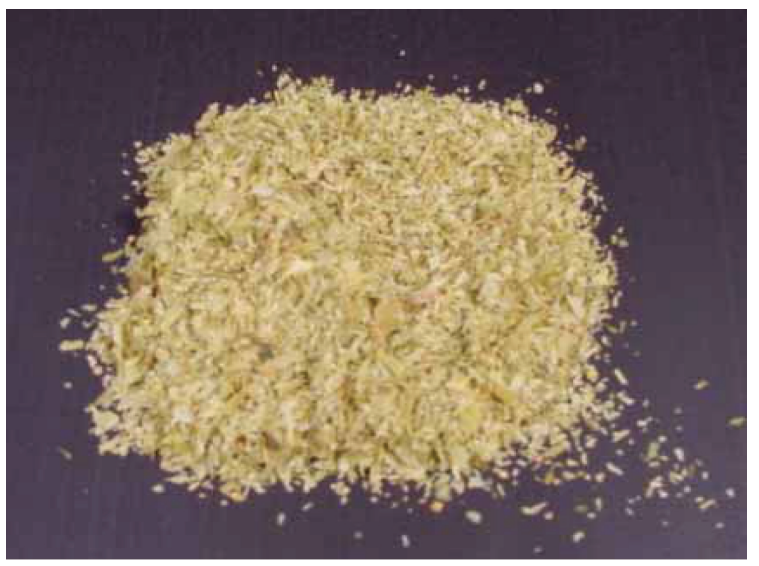

Fig. 2 The hop bract part discharged from beer breweries

experiment, $10 \mathrm{~g}$ of hop bract part and $200 \mathrm{dm}^{3}$ of extraction solvent were introduced into an erlenmeyer flask and shaken at $130 \mathrm{rpm}$. The extract solvent containing polyphenols and the hop bract part were then separated by suction filtration, after which distilled water was added to the solvent solution. Next, the alcohol in the extract solution was removed by rotary evaporation under reduced pressure. As a result of this treatment, an aqueous solution containing polyphenols was obtained. The aqueous solution was frozen using a freezedry machine resulting in a dry polyphenol extract, which was used in the extraction analysis. A pho-

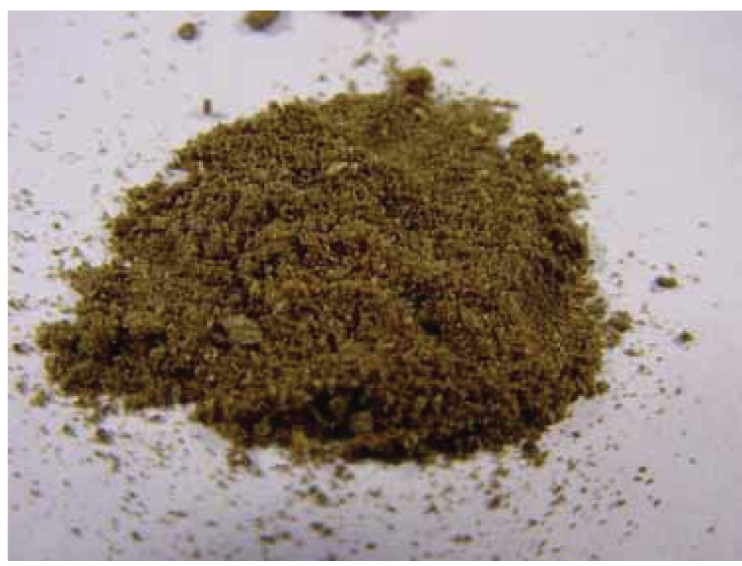

Fig. 3 The dry extract obtained from hop bract part

tograph of the collected dry extract, a highly hygroscopic green-brown powder, is shown in Figure 3.

\subsection{Analysis of the polyphenols in hop bract part (procyanidin and catechin)}

A colorimetric method using a phenolic hydroxyl reaction is widely used in the quantity analysis of polyphenols. The standard substance is selected according to the main kind of polyphenol included in the sample. The total amount of polyphenols in the sample is then calculated from the standard value (Saijou and Takeda, 1999). The analytical method used in this 


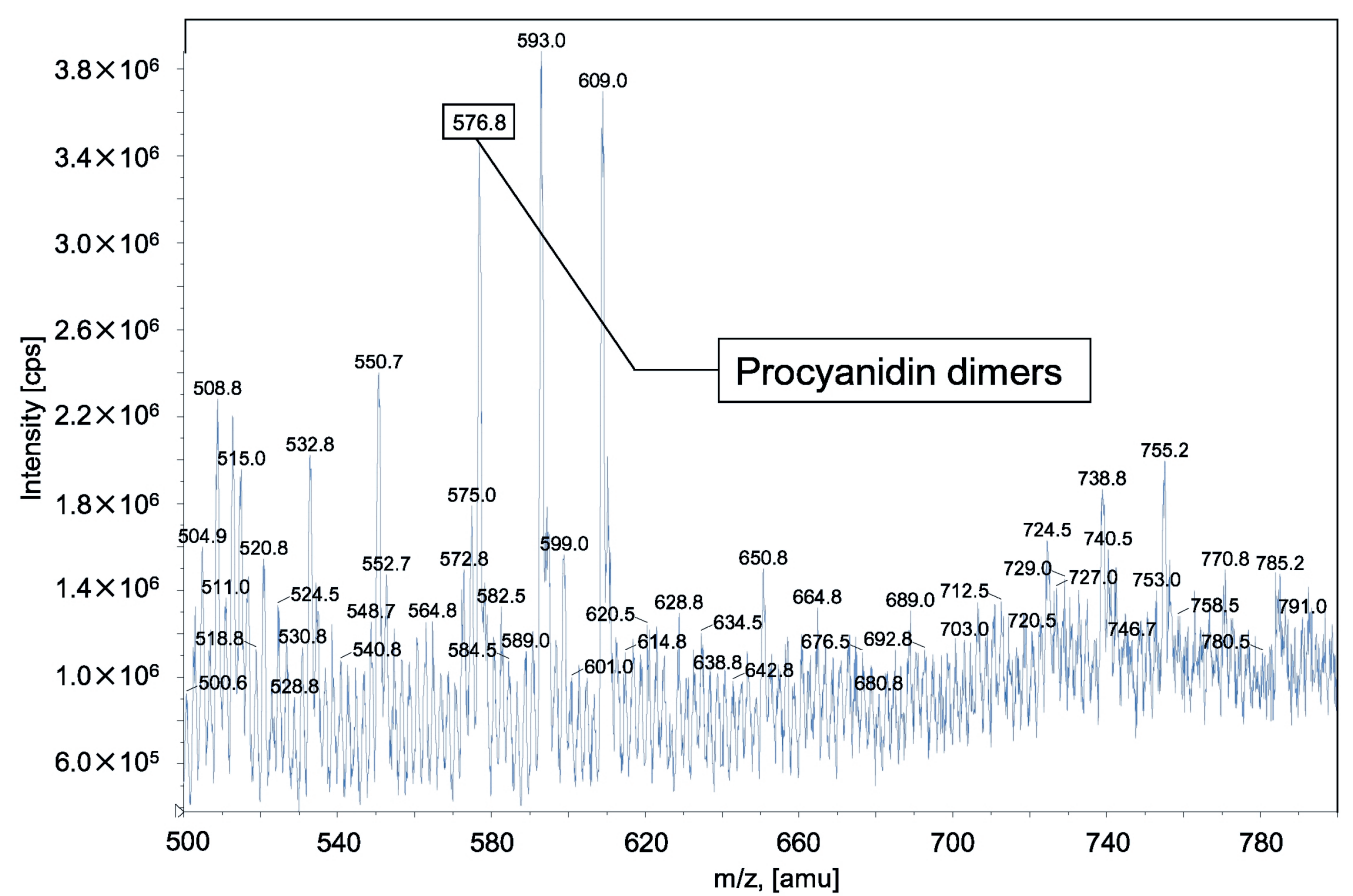

(a) Procyanidin dimers

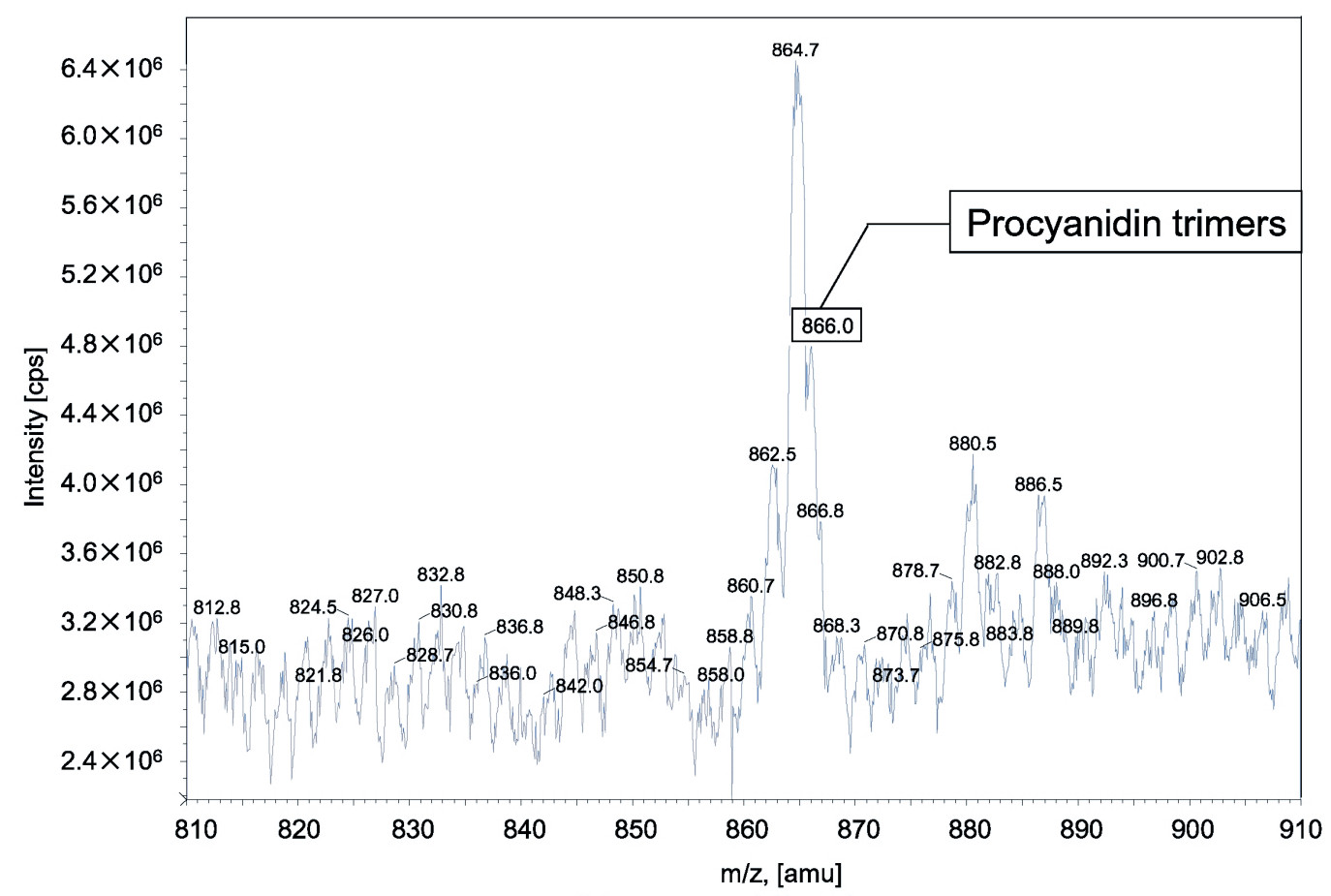

(b) Procyanidin trimers

Fig. 4 Chromatograms obtained from LC-MS analysis

research for catechin and procyanidin is shown below.

The amount of catechin was determined as a gallic acid equivalent, which represents the coloration yielded after combining galloyl component in catechin with iron. The total amount of catechin and procyanidin was determined using a vanillin assay (Iwasa and Torii, 1970); however, the obtained value is not an accurate calculated value of procyanidin because it includes the values of both catechin and procyanidin together.

The total amount of polyphenols was determined using the Folin-Denis method (Porter et al., 1986) with a commercially available phenol reagent. This method is suitable for measuring small amounts of polyphenol. The relative amount of procyanidin was calculated 


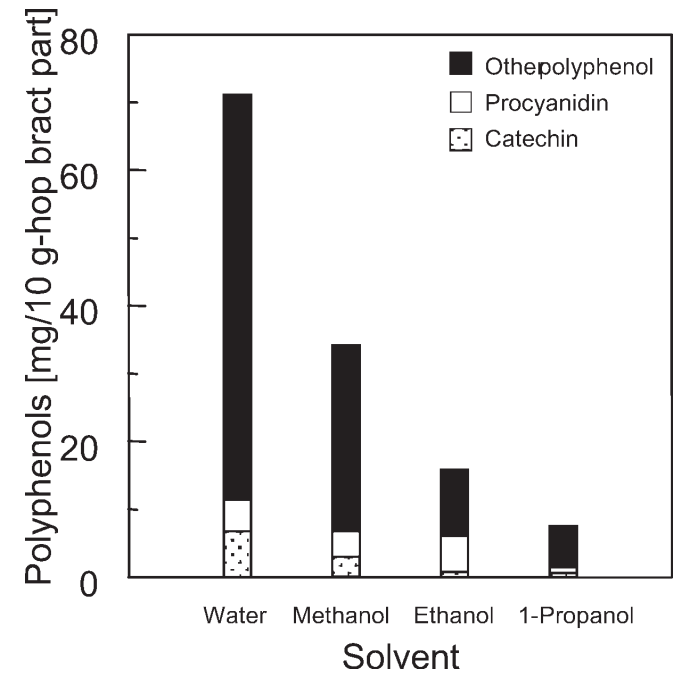

Fig. 5 Effect of various solvents on polyphenol extraction

using Porter's value. The coloration of cyanidin as a result of hydrolyzation of the highly polymerized procyanidin can also be measured using this method.

\section{Results and Discussion}

2.1 Analysis of procyanidin using liquid chromatography-mass spectrometry (LC-MS)

Extraction was carried out using ethanol as a solvent at an extraction temperature of $298 \mathrm{~K}$ and extraction time of $60 \mathrm{~min}$. The extract was analyzed by LCMS; the obtained chromatograms are shown in Figures 4(a) and (b). The results confirmed that procyanidin dimers (molar weight: 578.5 ) and trimers (molar weight: 866.9 ) are included in the dry extract obtained from the hop bract part.

\subsection{Extraction of polyphenol using various solvents}

Extraction of polyphenols was carried out using water, methanol, ethanol, and 1-propanol as the solvent, respectively. A comparison of the amount of polyphenol extracted with each solvent is shown in Figure 5. The extraction conditions are the extraction temperature of $298 \mathrm{~K}$ and the extraction time of 60 min. When water was used, the amount of polyphenol extracted was $70 \mathrm{mg}$ per $10 \mathrm{~g}$ of the hop bract part. With ethanol, the amount extracted was less than this, but the ratio of procyanidin was about $30 \%$. Of the solvents tested, ethanol had the highest selectivity for procyanidin. Differences in the amount of polyphenol extracted are closely related to the polarity of the solvent and polyphenol.

A comparison of the relative amount of procyanidin determined using Porter's value is shown in Figure 6. When methanol and ethanol were used as the solvent, the value of absorbance rose; that is, the relative amount increased. Methanol was shown to most effectively extract procyanidin. In this research,

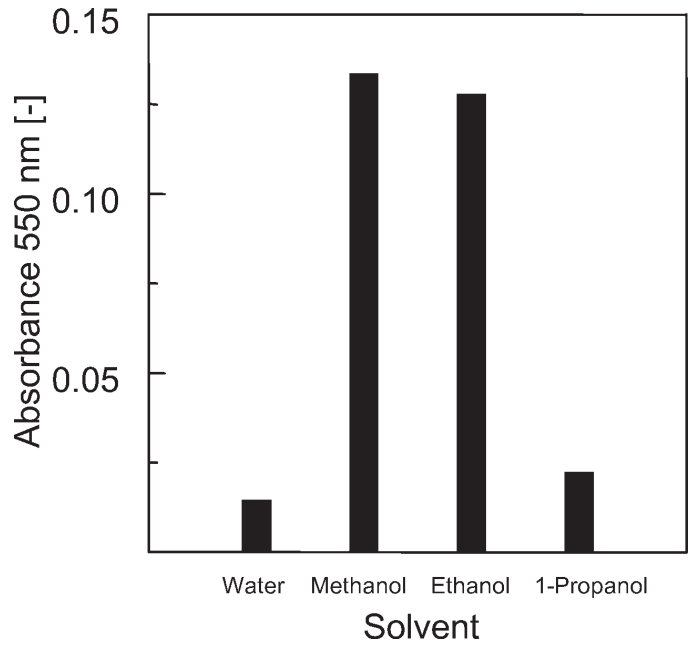

Fig. 6 The relative amount of procyanidin with each solvent

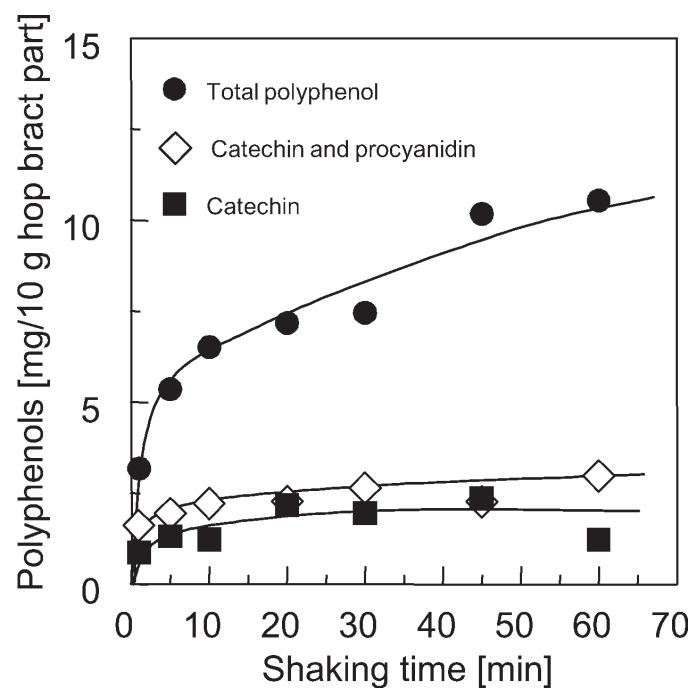

Fig. 7 Effect of shaking time on polyphenol extraction at $318 \mathrm{~K}$

ethanol or an aqueous ethanol solution that is harmless to the human body, was chosen as solvent from the viewpoint of practical use in the food manufacturing process, and the medicinal drug treatment. The following extraction experiments were therefore carried out using ethanol as a the solvent.

The relationship between the amount of polyphenol extracted and the extraction time is shown in Figure 7. The amount of catechin and procyanidin extracted increased with increasing extraction time, and it took about 10 min to reach equilibrium. An extraction time of $10 \mathrm{~min}$ was sufficient for equilibrium of catechin and procyanidin with all solvents used in this experiment.

The relationship between the amount of polyphenol extracted and the extraction temperature 
Table 1 Amount of polyphenols extracted from the hop bract part

\begin{tabular}{lccc}
\hline & $\begin{array}{c}\text { Total } \\
{[\mathrm{mg} / 10 \mathrm{~g} \text {-hop bract part] }}\end{array}$ & $\begin{array}{c}\text { Ratio to 10 g hop bract part } \\
\text { [wt\%] }\end{array}$ & $\begin{array}{c}\text { Ratio to dry extract } \\
\text { [wt\%] }\end{array}$ \\
\hline Dry extract & 620 & 6.2 & - \\
Total polyphenols & 26.0 & 0.26 & 4.19 \\
Procyanidin & 8.5 & 0.085 & 1.37 \\
Catechin & 1.5 & 0.015 & 0.24 \\
\hline
\end{tabular}

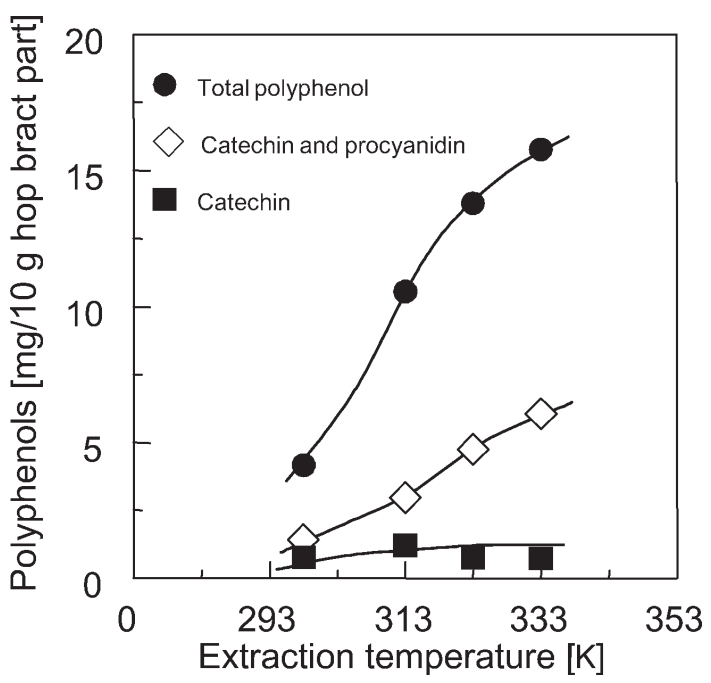

Fig. 8 Effect of temperature on polyphenol extraction for $60 \mathrm{~min}$

is shown in Figure 8. Because the boiling point of ethanol is $352 \mathrm{~K}$, the extraction temperature should be kept below $333 \mathrm{~K}$. The total amount of polyphenol extracted increased with increasing extraction temperature while the amount of catechin was approximately $0.73 \mathrm{mg}$ per $10 \mathrm{~g}$ of hop bract part at all temperatures tested. The amount of catechin contained in the hop bract part is thought to be minimal, and the amount extracted reached a maximum value at $333 \mathrm{~K}$. The total amount of catechin and procyanidin extracted was $6 \mathrm{mg}$ per $10 \mathrm{~g}$ of hop bract part at $333 \mathrm{~K}$, but the amount extracted did not reach a steady state at this temperature. Modification of the extraction technique is therefore required for optimization of the amount of procyanidin and catechin collected at 323-333 K.

The relationship between the amount of polyphenol extracted and the extraction frequency is shown in Figure 9. The total amount of polyphenol extracted decreased with increasing extraction frequency. The amount of catechin and procyanidin extracted greatly decreased from the third extraction onwards.

The total weight of the dry extract obtained from $10 \mathrm{~g}$ hop bract part, the total amount of polyphenols plus procyanidin and catechin extracted, and the ratio of each to the total weight of dry extract are shown in

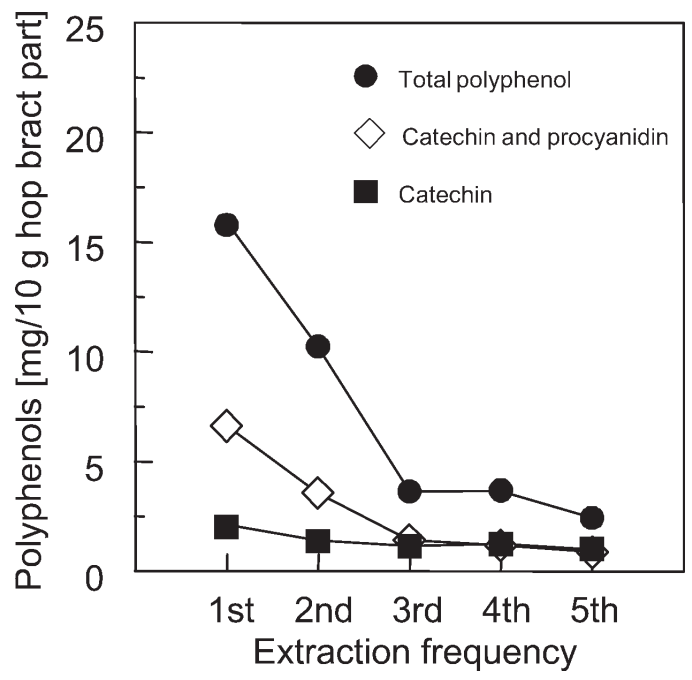

Fig. 9 Effect of extraction frequency on polyphenol extraction

Table 1. The results show that the amount of polyphenol extracted from the dry extract is notably condensed compared with the hop bract part.

The amount of polyphenol extracted from the hop bract part was compared with that from green and black tea using the extraction temperature of $333 \mathrm{~K}$, the extraction time of $60 \mathrm{~min}$, and an extraction frequency of both. A comparison of the amount of catechin extracted from each is shown in Figure 10(a). A comparison of the relative amount of procyanidin extracted from the hop bract part and both teas was also carried out using Porter's value; Figure 10(b) shows the absorbance obtained. The absorbance of procyanidin extracted from the hop bract part showed the highest value. Therefore, the amount of procyanidin with a high degree of polymerization (dimer or trimer) in the hop bract part was confirmed in this experiment. It was found that tea mainly contains low molecular weight monomer polyphenols such as catechin, while the hop bract part contains polymer polyphenols. Generally speaking, higher degrees of polymerization of catechin are thought to show its higher antioxidative property; therefore, the hop bract part discharged from beer breweries should be a valuable waste product. 


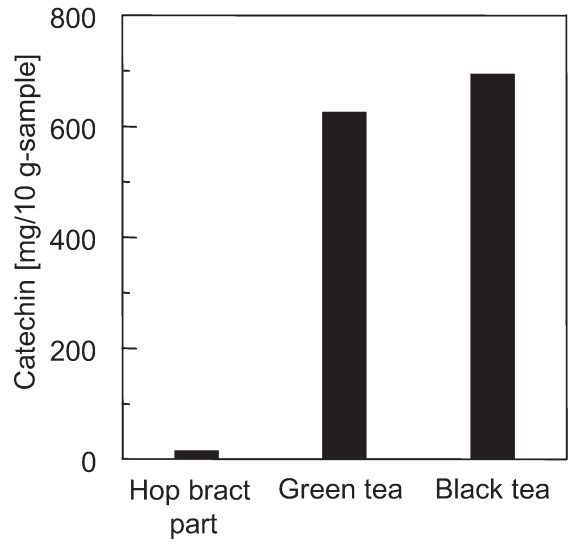

(a) Catechin

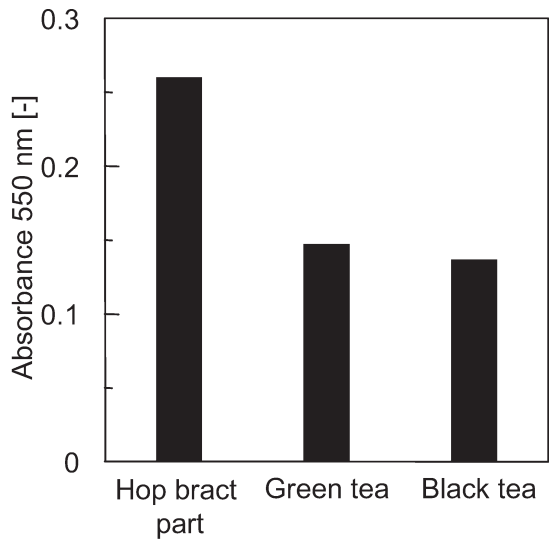

(b) The relative amount of procyanidin

Fig. 10 Comparison of the amount of polyphenol extracted from the hop bract part and green and black tea

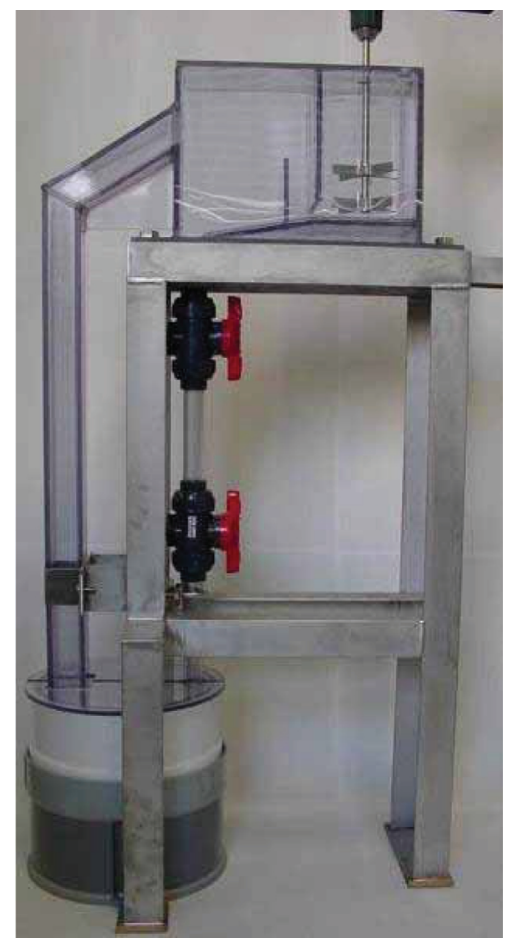

Fig. 11 Photograph of the solid-liquid extractor

\section{Continuous Solid-Liquid Extractor}

\subsection{Design of a continuous solid-liquid extractor}

A photograph and schematic diagram of the solidliquid extractor used in this research are shown in Figures 11 and 12. The mixer and the settler are made of transparent PVC (polyvinyl chloride) and have a volume of about 800 and $1400 \mathrm{dm}^{3}$, respectively. Crushed hop bract part and an extract solvent are mixed in the mixer unit using a mechanical stirrer; as a result of a downward flow formed by stirring with the mobile baffle plates (6 in Figure 12) the hop bract part and the extract solvent are mixed efficiently. They are continu-

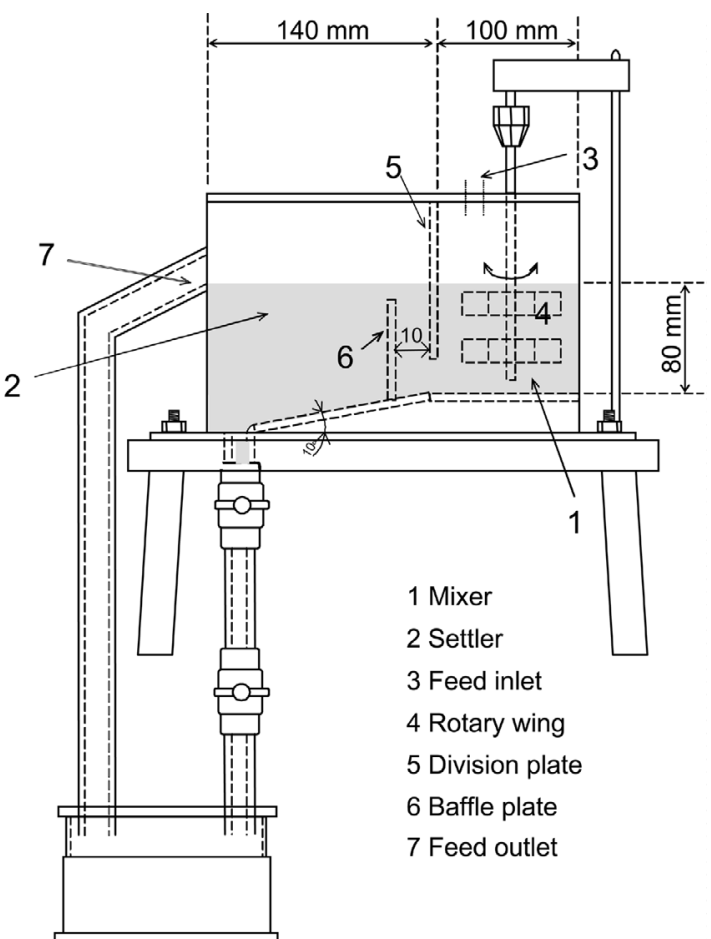

Fig. 12 Schematic diagram of the solid-liquid extractor

ously discharged from the mixer into the settler past a division wing then the solid hop bract part is collected as suspended material from the top of the unit.

\subsection{Extraction using the continuous solid-liquid extractor}

The solvent (ethanol) was introduced continuously into an extractor mixer (1) from a feed inlet (3) shown in Figure 12. Extraction treatment was conducted using $10 \mathrm{~g}$ of hop bract part at the rotation speed of $300 \mathrm{rpm}$, the solvent flow rate of $400 \mathrm{dm}^{3} / \mathrm{min}$ and operating time of $50 \mathrm{~min}$. In this experiment, only extract solvent was continuously flowed throughout the 
Table 2 Amount of polyphenol extracted using the solidliquid extractor

\begin{tabular}{lcc}
\hline & $\begin{array}{c}\text { Total } \\
{[\mathrm{mg} / 10 \text { g-hop bract part }]}\end{array}$ & $\begin{array}{c}\text { Extraction* } \\
{[\%]}\end{array}$ \\
\hline Catechin & 0.48 & 32.0 \\
Procyanidin & 1.8 & 21.1 \\
Total polyphenol & 8.8 & 33.8 \\
\hline
\end{tabular}

* Ratio to the total amount of polyphenols obtained by batch operation

extractor; hop bract part was supplied only when required for extraction. Polyphenole concentration in the outflow solvent was measured at a feed outlet (7) with constant intervals of $1 \mathrm{~min}$. Table 2 shows the amount of polyphenol contained in the outflow solvent; the percentage extraction shows the ratio to the total amount of polyphenols obtained from the hop bract part by batch operation. The percentage extraction of total polyphenol, procyanidin, and catechin using the solidliquid extractor were $33.8,21.1$, and $32.0 \%$ of that in batch operation, respectively. Three or four stages of the presented mixer-settler extractor are needed in order to separate the total amount of polyphenol in the hop bract part.

\section{Conclusions}

In this study, it was found that procyanidin dimers and trimers are included in the dry extract obtained from the hop bract part. When ethanol was used as the extract solvent, about $30 \%$ of the total extracted polyphenols was procyanidin, showing that ethanol has the highest selectivity for procyanidin. An extraction time of 10 min was sufficient for equilibrium of catechin and procyanidin with all solvents used in this experiment. The effect of extraction for procyanidin and catechin on temperature were examined in the temperature rang from 323 to $333 \mathrm{~K}$. The total amount of catechin extracted reached a maximum at an extraction temperature of $333 \mathrm{~K}$. The amount of polyphenol extracted from the hop bract part was compared with that from green and black tea. It was found that tea mainly contains low molecular weight monomer polyphenols such as catechin, while hop bract part contains polymer polyphenols. The hop bract part from beer breweries discharge catechin, while it contains polymers, which makes the hop bract part a valuable waste product.

The mixer-seller extractor designed and manufactured to extract the polyphenols contained in the hop bract part was tested under several experimental conditions. The percentage extraction was deduced to be $20-30 \%$ of that in a batch operation. Three stages of the presented mixersettler extractor are needed in order to separate the total amount of polyphenol in the hop bract part.

\section{Acknowledgment}

This research was supported by the Research and Development Organization of Industry-University Cooperation from the Ministry of Education, Culture, Sports, Science and Technology of Japan.

\section{Literature Cited}

Ariga, T., I. Koshiyama and D. Fukushima; "Antioxidative Properties of Procyanidins B-1 and B-3 from Azuki Beans in Aqueous Systems," Agric. Biol. Chem., 52, 2717-2722 (1988)

Guyot, S., N. Marnet, D. Laraba, P. Sanoner and J. F. Drilleau; "Reversed-Phase HPLC Following Thiolysis for Quantitative Estimation and Characterization of the Four Main Classes of Phenolic Compounds in Different Tissue Zones of a French Cider Apple Variety," J. Agric. Food Chen., 46, 1698-1705 (1998)

Iwasa, K. and H. Torii; "Average Content and Extraction Rate of Mineral Element in Tea," Chagyou Kenkyuu Houkoku, 19, 104108 (1970)

Kanner, J, E. Frankel, R. Granit, B. German and J. E. Kinsella; "Natural Antioxidants in Grapes and Wines," Agric. Biol. Chem., 42, 64-69 (1994)

Porter, L. J., L. N. Hrstich and B. G. Chan; "The Conversion of Procyanidins and Prodelphinidins to Cyanidin and Delphindin," Phytochemistry, 25, 223-230 (1986)

Saijou, R. and Y. Takeda; "HPLC Analysis of Catechins in Various Kinds of Green Teas Produced in Japan and Abroad," Nippon Shokuhin Kagaku Kougaku Kaishi, 46, 138-147 (1999)

Suematsu, S., Y. Hisanobu, H. Saigou, R. Matsuda and Y. Komatsu; "Studies on Preservation of Constituents in Canned Drinks. Part V. A New Extraction Procedure for Determination of Caffeine and Catechins in Green Tea," Nippon Shokuhin Kagaku Kougaku Kaishi, 42, 419-424 (1995)

Yamamoto, H., C. Nagano, F. Takeuchi and J. Shibata; "Extraction of Polyphenols in Hop Waste Discharged from Beer Breweries," 7th International Conference of Separation Science and Technology between Korea and Japan, pp. 257-263, Yeongju, Korea (2005a)

Yamamoto, H., F. Takeuchi, C. Nagano, A. Sasaki and J. Shibata; "Extraction Properties of Flabonoids in Bean Cake Disposed from Soy Sauce Manufacturing Process," 7th International Conference of Separation Science and Technology between Korea and Japan, pp. 242-248, Yeongju, Korea (2005b) 